\title{
Harnessing the Power of Molecular Imaging for Precision Medicine
}

\author{
Emily B. Ehlerding ${ }^{1}$ and Weibo Cai ${ }^{1,2}$ \\ ${ }^{1}$ Departments of Radiology and Medical Physics, University of Wisconsin-Madison, Madison, Wisconsin; and ${ }^{2}$ University of \\ Wisconsin Carbone Cancer Center, Madison, Wisconsin
}

$\mathbf{T}$ he field of molecular imaging has witnessed tremendous expansion over the last decade (1). A wide variety of molecular imaging agents (e.g., radiolabeled, fluorescently labeled, and nanoparticlebased) have been developed and optimized for preclinical and clinical investigation. Now is the prime time to reap the fruit of such tremendous development in the field via the use of multiparametric molecular imaging to monitor the efficacy of targeted therapies and guide the rational design of more efficacious combination therapy. In this issue of The Journal of Nuclear Medicine, Gao et al. from Peking University Health Science Center reported the elegant use of multimodality, multiparametric molecular imaging to interrogate the biologic effects of a drug in a mouse tumor model, which revealed valuable insights into the mechanisms of the drug action and led to the design of a combination therapy strategy with significantly improved efficacy (2).

\section{See page 321}

The tumor model was generated using a human non-small cell lung cancer cell line stably transfected with firefly luciferase (i.e., A549fLuc), which allowed for convenient measurement of tumor growth via bioluminescence imaging (BLI). Such measurement by BLI can complement and cross-validate traditional tumor size measurement obtained via a digital caliper. The 3 imaging agents used in this study span PET, SPECT, and near-infrared fluorescence (NIRF): ${ }^{18} \mathrm{~F}-\mathrm{FDG}$, which is used daily in clinical oncology and reflects tumor metabolic activity via PET (3); ${ }^{99 m}$ Tc-3PRGD2, which was developed in their laboratory for SPECT imaging of integrin $\alpha_{\mathrm{v}} \beta_{3}$ expression, currently in clinical investigation (4); and the NIRF probe DyLight755ranibizumab, which is specific for human vascular endothelial growth factor (VEGF) but does not bind to murine VEGF (5). All these agents have been extensively studied and rigorously validated previously.

In the initial study, the investigators tested a single-agent treatment using dasatinib, a Food and Drug Administration-approved drug that inhibits multiple tyrosine kinases such as the BCR-Abl and Src family kinases, c-Kit, and ephrin receptors, among others (6). After mice

Received Sep. 3, 2015; revision accepted Sep. 4, 2015

For correspondence or reprints contact: Weibo Cai, University of Wisconsin-Madison, Rm. 7137, 1111 Highland Ave., Madison, WI 53705

E-mail: wcai@uwhealth.org

Published online Sep. 24, 2015.

COPYRIGHT (C 2016 by the Society of Nuclear Medicine and Molecular Imaging, Inc.

DOI: $10.2967 /$ jnumed.115.166199 bearing A549-fLuc tumors were treated with dasatinib for 6 consecutive days, neither BLI nor ${ }^{18} \mathrm{~F}$-FDG PET showed any statistically meaningful difference between the control and treated groups. Ex vivo histologic studies corroborated the in vivo finding, confirming the lack of antitumor activity when tumor metabolism or proliferation was selected as the endpoint. Because BLI and ${ }^{18}$ F-FDG PET are routinely used to monitor the therapeutic efficacy of various treatments in preclinical studies, one may come to the conclusion at this point that these results merely indicate a failed pilot study.

However, the 2 molecular imaging agents used in this study came to the rescue. Noninvasive imaging using ${ }^{99 m}$ Tc-3PRGD2 and DyLight755-ranibizumab both revealed that the dasatinib-treated group showed significantly lower signal in the tumor, suggesting that although there is no significant effect for dasatinib alone in reducing tumor metabolism or proliferation, there are indeed important biologic effects taking place after such single-agent treatment, namely antiangiogenic activity. After extensive ex vivo experiments to decipher this finding, it was concluded that the mechanisms at work in these 2 imaging studies do have subtle differences, even though both studies showed a decrease in signal and both imaging targets (integrin $\alpha_{v} \beta_{3}$ and VEGF) are closely related to tumor angiogenesis.

The decrease in signal from DyLight755-ranibizumab can be readily explained: because it is specific for human VEGF, the reduced signal can be attributed to lower production of VEGF by the tumor cells. Of note, VEGF has many isoforms, most of which are cellmembrane bound and only VEGF $_{121}$ is freely diffusible. Therefore, it is likely that the NIRF signal is mostly from membrane-bound VEGF isoforms (e.g., $\mathrm{VEGF}_{165}, \mathrm{VEGF}_{189}$, and $\mathrm{VEGF}_{206}$ ). Another potential contributing factor to the NIRF imaging results could be that dasatinib reduces the solid tumor penetration of DyLight755-ranibizumab via inhibition of tumor angiogenesis, an effect that may deserve to be studied in the future. Such effect is generally more profound for antibody-based agents (e.g., DyLight755-ranibizumab) than lowermolecular-weight agents such as ${ }^{99 m}$ Tc-3PRGD2.

The explanation for decreased tumor uptake of ${ }^{99 m}$ Tc-3PRGD2 in the dasatinib-treated group requires much more investigation and elaboration. The data presented in the article suggested that there is no significant decrease in integrin $\alpha_{v} \beta_{3}$ expression level on the tumor cells based on staining with an antibody specific for human $\alpha_{v} \beta_{3}$, LM609. However, the expression of murine integrin $\alpha_{v} \beta_{3}$ on the tumor vasculature was significantly abrogated after dasatinib treatment. Furthermore, it was also confirmed that dasatinib caused significant inactivation of integrin $\alpha_{v} \beta_{3}$, leading to much weaker binding

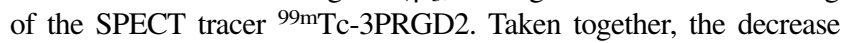
of ${ }^{99 m}$ Tc-3PRGD2 uptake in the dasatinib-treated group can be attributed to both of the following factors: decreased murine integrin 
$\alpha_{v} \beta_{3}$ expression on the tumor vasculature and inactivation of integrin $\alpha_{v} \beta_{3}$ by dasatinib. It is highly likely that the latter happened on both A549-fLuc cells and tumor vasculature endothelial cells, expressing human and murine integrin $\alpha_{\mathrm{v}} \beta_{3}$, respectively, although it is difficult to tease this aspect out due to the lack of antibodies that can differentiate activated integrin $\alpha_{\mathrm{v}} \beta_{3}$ from its inactivated form.

Thanks to the molecular specificity of the imaging agents, which provided exquisite information, it is logical to hypothesize that adding another agent that can kill tumor cells (because dasatinib is effective in inhibiting tumor angiogenesis) may lead to enhanced therapeutic effect. Indeed, when docetaxel was added to the treatment regimen every other day, the A549-fLuc tumor growth was significantly inhibited. Because the combination therapy of dasatinib and docetaxel targets both tumor cells and tumor vasculature, all subsequent imaging studies showed significantly reduced tumor uptake in the treated group, corroborated by histologic examination, and confirmed the therapeutic efficacy.

This work is an elegant example of a rationally designed, multimodal, multiparametric molecular imaging study that harnessed the tremendous power of molecular imaging and turned a failed pilot study into a success story. If traditional anatomic imaging techniques such as CT or MR imaging were used to solely measure the tumor size, no biologic/functional information would have been obtained. Even ${ }^{18} \mathrm{~F}-\mathrm{FDG}$, a PET tracer that is used daily in the clinic, did not show any therapeutic response because dasatinib alone is not effective in shutting down the tumor metabolism or shrinking the tumor at the dose used. The addition of molecular imaging agents to treatment planning is critical for a more detailed interrogation of the various biologic pathways affected by the drugs.

Several research avenues can be pursued in future studies. First, further optimization of the combination therapy protocol will be needed. In this work, although the combination of dasatinib and docetaxel significantly slowed tumor growth, the tumor size was not reduced. Optimization of the dosing, as well as other drug combinations, may lead to better efficacy, which could eventually eliminate the tumor. Other treatment modalities may also be explored such as targeted radiotherapy and nanoplatforms (7) that can more efficiently deliver drugs to the tumor. Second, most tyrosine kinase inhibitors are dirty inhibitors because they bind to many kinases and inhibit several signaling pathways. In this study, the focus was on Src, which plays a critical role in integrin signaling and activation, and integrin, which has an intricate relationship with the VEGF/VEGF receptor pathway (8). Dasatinib binds to many other targets as well, such as c-Kit and ephrin receptors. Thus, imaging of other related pathways may reveal additional venues for future combination therapy. Third, the quantitation for ${ }^{18} \mathrm{~F}$ FDG PET was presented as the standard percentage injected dose per gram of tissue. However, the uptake of ${ }^{99 m}$ Tc-3PRGD2 was presented as tumor-to-muscle ratio, which is not ideal. Although this may be due to the traditional qualitative nature of SPECT scanners, quantification with SPECT is becoming more feasible (9), and additional quantitative data may provide even more insights into the drug mechanisms. Fourth, because there are numerous new molecularly targeted drugs under development or commercially available, possible future research avenues in this area are virtually unlimited.

Currently, there are many tracers available for the imaging of integrin $\alpha_{\mathrm{v}} \beta_{3}$ in the clinic, including both PET and SPECT agents (10-13). For imaging of VEGF, ${ }^{89} \mathrm{Zr}$-labeled antibodies have also entered clinical investigation (14). Therefore, similar studies can be readily performed in the clinic to better tailor the treatment for individual cancer patients to achieve more personalized and precise treatments. To realize the long-term goal of precision medicine, multidisciplinary teams need to work together in a concerted manner, which also mandates the regulatory agencies to lower the barriers for clinical translation of promising PET/SPECT tracers as well as optical agents for image-guided tumor resection. To date, clinical development and investigation of these highly powerful molecular imaging agents is primarily limited to major medical centers and institutions, inaccessible to most researchers, clinicians, and patients.

A few years ago, many preclinical treatment studies were relying on simple digital caliper measurements of tumor size and sacrificing large numbers of animals at different time points for various ex vivo studies such as Western blot analyses, histology, and others. With the wider availability of better and more powerful tools, in terms of both imaging agents and instrumentation, more complex studies such as this are becoming increasingly more common from major research groups and medical centers globally. Clearly, routine studies such as this in the clinical setting are still quite challenging, but the field of molecular imaging has matured and is steadily moving in the right direction toward harnessing the tremendous power of molecular imaging for more effective therapies. This progress, in combination with the advances in other disciplines such as ex vivo sensing, genomic/proteomic/metabolomic tools, and immunotherapy, has allowed the dawning era of personalized and precision medicine for cancer patient management to be on us today.

\section{DISCLOSURE}

This work is supported, in part, by the University of WisconsinMadison, the National Institutes of Health (NIBIB/NCI 1R01CA169365, P30CA014520, T32GM008505), and the American Cancer Society (125246-RSG-13-099-01-CCE). No other potential conflict of interest relevant to this article was reported.

\section{REFERENCES}

1. James ML, Gambhir SS. A molecular imaging primer: modalities, imaging agents, and applications. Physiol Rev. 2012;92:897-965.

2. Gao L, Liu H, Sun X, et al. Molecular imaging of post-Src-inhibition tumor signatures for guiding dasatinib combination therapy. J Nucl Med. 2016;57:321-326.

3. Gambhir SS, Czernin J, Schwimmer J, Silverman DH, Coleman RE, Phelps ME. A tabulated summary of the FDG PET literature. J Nucl Med. 2001;42:1S-93S.

4. Zhu Z, Miao W, Li Q, et al. ${ }^{99 \mathrm{~m} T c-3 P R G D 2}$ for integrin receptor imaging of lung cancer: a multicenter study. $J$ Nucl Med. 2012;53:716-722.

5. Ma T, Liu H, Sun X, et al. Serial in vivo imaging using a fluorescence probe allows identification of tumor early response to cetuximab immunotherapy. Mol Pharm. 2015;12:10-17.

6. Shah NP, Tran C, Lee FY, Chen P, Norris D, Sawyers CL. Overriding imatinib resistance with a novel ABL kinase inhibitor. Science. 2004;305:399-401.

7. Chen F, Ehlerding EB, Cai W. Theranostic nanoparticles. J Nucl Med. 2014;55: 1919-1922.

8. De S, Razorenova O, McCabe NP, O’Toole T, Qin J, Byzova TV. VEGF-integrin interplay controls tumor growth and vascularization. Proc Natl Acad Sci USA. 2005;102:7589-7594.

9. Bailey DL, Willowson KP. An evidence-based review of quantitative SPECT imaging and potential clinical applications. $J$ Nucl Med. 2013;54:83-89.

10. Liu Z, Wang F. Development of RGD-based radiotracers for tumor imaging and therapy: translating from bench to bedside. Curr Mol Med. 2013;13:1487-1505.

11. Sun Y, Zeng Y, Zhu Y, et al. Application of ${ }^{68}$ Ga-PRGD2 PET/CT for $\alpha_{v} \beta_{3}$-integrin imaging of myocardial infarction and stroke. Theranostics. 2014;4:778-786.

12. Wan W, Guo N, Pan D, et al. First experience of ${ }^{18} \mathrm{~F}$-alfatide in lung cancer patients using a new lyophilized kit for rapid radiofluorination. J Nucl Med. 2013;54:691-698.

13. Iagaru A, Mosci C, Mittra E, et al. Glioblastoma multiforme recurrence: an exploratory study of ${ }^{18} \mathrm{~F}$ FPPRGD 2 PET/CT. Radiology. May 12, 2015 [Epub ahead of print].

14. Oosting SF, Brouwers AH, van Es SC, et al. ${ }^{89} \mathrm{Zr}$-bevacizumab PET visualizes heterogeneous tracer accumulation in tumor lesions of renal cell carcinoma patients and differential effects of antiangiogenic treatment. $J$ Nucl Med. 2015;56:63-69. 